\title{
FUZZY BASED DATA CONGLOMERATION MODEL IN MULTI SENSOR FOR OPTIMIZING AQUAFARM
}

\section{G. SHAHANA, VINEETA DEEPTHI \& I. JOHNSI STELLA}

Department of Electronics and Communication Engineering, St. Joseph's College Engineering,

OMR, Chennai-600 119, Tamil Nadu

\begin{abstract}
The success of real time system especially aquaculture and allied projects largely depend upon the quality of the site selected for the projects. The selection of best site in aquaculture system is not formulated just from one variable alone but from multiple variables. In the emerging technology era, these variables are collected using sensor-based network systems, wireless sensor networks (WSNs). Due to the environmental characteristics, limited power and processing capabilities of WSNs, it is essential to find techniques that improve the flow of information in the network. Data conglomeration can be used to minimize the amount of information flowing, and the energy spent during sensing, processing and communication operations in the network. In this backdrop, fuzzy logic based data conglomeration model in multi sensor was developed for optimizing aquafarms. This leads to effective utilization of various resources like water, land, infrastructure facilities, support and inputs and hence becomes a cost effective system for the expected yield. The results show that the application is correct and reasonable and enables the user to precisely acquire the culture requirement information. The proposed models provide tools that will help the planners and the decision-makers to make choices regarding the development of aquaculture farming practices.
\end{abstract}

KEYWORDS: Wireless Sensor Networks, Fuzzy Logic, Mamdani Fuzzy Inference System, Aquaculture, Optimization.

Received: Dec 20, 2020; Accepted: Dec 30, 2020; Published: Dec 30, 2020; Paper Id.: IJMCARDEC20209

\section{INTRODUCTION}

Wireless sensor networks have limited computing power and limited memory and battery capacity, this will increase the complexity for the application developer and many programs that have a tightly coupled network protocol. In addition, the success of both real-time systems, particularly aquaculture and their project allies, is highly dependent on the quality of the site chosen for the project. The success of aqua-cultural projects, but the negative environmental impact depends largely on the quality of the site chosen for the project (Boyd and Clay, 1998), and also depends on the definition but also the development of fisheries based on several factors (McKindsey et al., 2006).

New technologies are emerging, and this data is collected using sensor networks, wireless sensor networks (WSNs). The main environmental functions, limited forces and e ' capabilities of WSNs, it is important to find ways to improve information flows in the network. Information was given about the model based on the Mamdani fuzzy logic system used to reduce traffic and improve the performance of sensor networks. A data acquisition protocol that is designed to eliminate unnecessary data transmission and thus increase the service life of the limited power of the wireless sensor network.

But previous studies have shown that a number of factors, like soil and water quality, are difficult to 
handle using Conventional Logical logic (Crisp), which is usually the use of geographic information systems (GIS) and remote sensing (DZ) technology (Tarunamulia, 2008). The latest development of fuzzy logic technology has suggested an alternative way to deal with the shortcomings of this practice in the context of real-world complexity (Tarunamulia, 2008). Fuzzy systems have successfully applied classification, modeling, control, and a wide range of applications (Singh et al., 2006). Apply fuzzy "if-then" rules and greatly improve the interpretability of the result and give you more insight into the structure theory and decision-making process (st John et al., 2003).

In view of all the above aspects, fuzzy logic based data conglomeration model in multi sensor system was developed for optimizing aquafarms. This leads to effective utilization of various resources like water, land, infrastructure facilities, support and inputs and hence becomes a cost effective system for the expected yield.

\section{FUZZY MODEL DEVELOPMENT}

\section{Variables and Datasets}

In this study, six variables like $\mathrm{pH}(\mathrm{pH})$, dissolved oxygen (DO), duzluq (S), open (Oh), and organic carbon (OC) were selected by reviewing the literature (e.g., CIBA, 2018. Mahalakshmi, and Ganesan, 2009), as well as consulting with experts who have all worked in the field of fisheries. Assessment of suitable identified variables taken from different texts, defined as amended, India environment, based on the opinion of an aquaculture expert.

The suitability assessment is issued into three categories: (1) where appropriate, assessments show that the relevant variable is advantageous, intended for use, and is not an unusual design, construction, management, or service that is required at the designated location, for the purposes of uses. (2) Soft (very good), estimates show that the corresponding variable is beneficial for its intended use and special attention will be required in the designated place for uses. (3) an inadequate assessment shows that the relevant variable is detrimental to the intended use and that the designated website may be useful for a particular purpose beyond large circulation, as well as changes in design, management, and or maintenance, but using the website with one or more unjustified restrictions may be ineffective. The identified variables and estimates, as well as the defined goal \# 1, are shown in the table. $\mathrm{PH}(\mathrm{PH})$, dissolved oxygen (DO), duzluq (S), open $(\mathrm{Oh})$, organic carbon $(\mathrm{OC})$, and the relevant data used in this case were achieved by the ICAR Institute, Chennai, Tamil Nadu.

\section{Proposed Fuzzy Model}

In the proposed fuzzy model, both the antecedent and consequent parts were expressed by the experts in fuzzy statements using linguistic variables such as suitable, moderately suitable 1 , moderately suitable 2 , unsuitable 1 or unsuitable 2 . Therefore a Mamdani inference system was used to develop this model. Schematic representation of the proposed fuzzy model is shown in Figure 1. 


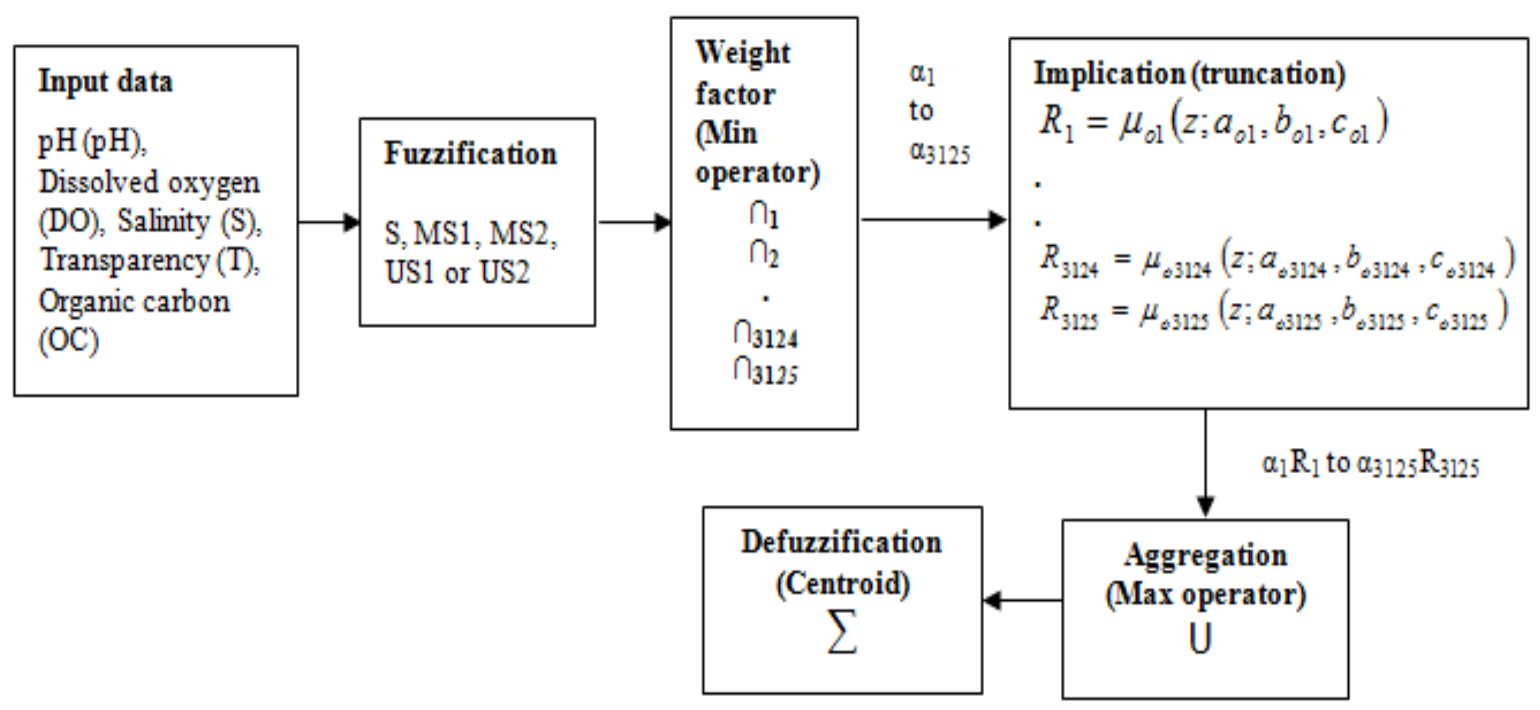

Figure 1: Schematic Representation of Proposed Fuzzy Model

\subsubsection{Fuzzy Sets and Membership Functions}

Fuzzy logic is a process of mapping an input space into an output space using membership functions and linguistically specified rules. In the proposed fuzzy model, five inputs and one output were used to classify the optimal location in aquaculture. The inputs were $\mathrm{pH}(\mathrm{pH})$, Dissolved oxygen (DO), Salinity (S), Transparency (T), Organic carbon (OC) represented by $\mathrm{X}$ and output was Aqua Farm Classification (AFC) represented by Z. Mathematically this can be represented as $Z=F(X)$. This model of both input and output variables is divided into five linguistic variables, namely appropriate (S), soft fitting1 (MS1), soft fitting2 (MS2), unusable1 (US1) or unusable2 (US2). After separating the variables, a membership function is defined for each of the linguistic variables. In this project, based on the experience and knowledge of experts, Pedrycz, 2001), trapezoidal and triangular MFS and their range were selected for the input and output variables, respectively. Trapezoidal and triangular MFs were defined by

$$
\mu_{i j}(x)=f\left(z ; a_{i j} ; b_{i j} ; c_{i j} ; d_{i j}\right)= \begin{cases}0 & x \leq a_{i j} \\ \frac{x-a_{i j}}{b-a} & a_{i j} \leq x \leq b_{i j} \\ 1 & b_{i j} \leq x \leq c_{i j} \\ \frac{d_{i j}-x}{d_{i j}-c_{i j}} & c_{i j} \leq x \leq d_{i j} \\ 0 & d_{i j} \leq z_{i j}\end{cases}
$$

for $(\mathrm{i}=1$ to $5 ; \mathrm{j}=1$ to 5$) \mathrm{x}=(\mathrm{pH}, \mathrm{DO}, \mathrm{S}, \mathrm{T}, \mathrm{OC})$ where $\mu_{i j}$ represent the $\mathrm{j}^{\text {th }} \mathrm{MF}$ of the $\mathrm{i}^{\text {th }}$ input; $a_{i j}, b_{i j}$, $c_{i j}, d_{i j}$ are the parameters that represent the shapes of the input MF 


$$
\mu_{o j}(z)=\operatorname{Tri}\left(z ; a_{o j} ; b_{o j} ; c_{o j}\right)=\left\{\begin{array}{cc}
0 & z \leq a_{o j} \\
\frac{z-a_{o j}}{b_{o j}-a_{o j}} & a_{o j} \leq z \leq b_{o j} \\
\frac{c_{o j}-z}{c_{o j}-b_{o j}} & b_{o j} \leq z \leq c_{o j} \\
0 & c_{o j} \leq z
\end{array}\right.
$$

for $(\mathrm{o}=1 ; \mathrm{j}=1,2,3) \mathrm{z}=(\mathrm{AFC})$ where $\mu_{o j}$ represent the $\mathrm{j}^{\text {th }}$ output MF; $a_{i j}, b_{i j}, c_{i j}$ are the parameters that represent the shapes of the output MF. Table 2 and 3 present the linguistic variables for each of the input and output variables respectively.

\subsubsection{Fuzzy Rule Base}

Many researchers have investigated techniques for determining rules such as fuzzy classifier, neural network, genetic algorithm and expert knowledge (Mazloumzadeh et al., 2010). In this model, experts' knowledge and order of importance of the variables were used for the construction of fuzzy rule base (Center and Verma, 1998). The proposed model has 5 X 5 X 5 X 5 X $5=3125$ rules based on the five input variables and their corresponding linguistic variables. These if-then rules were collated with AND operator because all the input variables must be captured simultaneously and applied in decision making by fuzzy logic for classification. The fuzzy rule base for the proposed model was defined as follows:

1. if ( $\mathrm{pH}$ is $\mu_{11}$ ) and (DO is $\left.\mu_{21}\right)$ and (S is $\left.\mu_{31}\right)$ and ( $\mathrm{T}$ is $\mu_{41}$ ) and (OC is $\left.\mu_{51}\right)$ then

$$
R_{1}=\mu_{o 1}\left(z ; a_{o 1}, b_{o 1}, c_{o 1}\right)
$$

2. if ( $\mathrm{pH}$ is $\mu_{11}$ ) and (DO is $\mu_{21}$ ) and (S is $\left.\mu_{31}\right)$ and ( $\mathrm{T}$ is $\mu_{41}$ ) and (OC is $\mu_{51}$ ) then $R_{2}=\mu_{o 2}\left(z ; a_{o 2}, b_{o 2}, c_{o 2}\right)$

if (pH is $\mu_{13}$ ) and (DO is $\left.\mu_{23}\right)$ and (S is $\mu_{33}$ ) and ( $\mathrm{T}$ is $\mu_{43}$ ) and (OC is $\left.\mu_{53}\right)$ then $R_{3125}=\mu_{o 3125}\left(z ; a_{o 3125}, b_{o 3125}, c_{o 3125}\right)$

where $\mu_{i j}$ is the $\mathrm{j}^{\text {th }}$ MF of the $\mathrm{i}^{\text {th }}$ input; $\mu_{o k}$ is the $\mathrm{k}^{\text {th }}$ output MF; $R_{k}$ is the output of the $\mathrm{k}^{\text {th }}$ rule; $a_{o k}, b_{o k}, c_{o k}$ are the parameters that represent the shapes of the output MFs.

\subsubsection{Operating Mechanism of the Proposed Model}

The proposed model consists of five operating mechanisms named as fuzzification, calculation of weight factor, implication, aggregation, and defuzzification (Fig. 1). 


\section{Step 1: Fuzzification}

At this point, the exact input becomes a fuzzy input, an MFS input. This model can also say that in each class, input variables overlapped with neighboring classes, because decision-making distributed another input to the class. (Fig. 2(a \& b)). Furthermore, to make the output clear and unbiased, the symmetrical, non-overlapping equal-size membership functions were used for the output variable (Fig. 3).

\section{Step 2: Calculation of Weight Factor}

In this step, the weighting factor of each rule was calculated. This was computed by first converting the input values to fuzzy membership values by using the input MFs in step1 and then applying the "AND" operator to these membership values. The "AND" operator corresponds to the minimum of input membership values.

\section{Step 3: Implication}

For classification applications, implication is carried out by either truncation or scaling. Truncation is done by chopping off the output MF, while scaling is done by compressing the function. In this model, truncation implication was used which is one of the most widely used implication in applications of fuzzy logic.

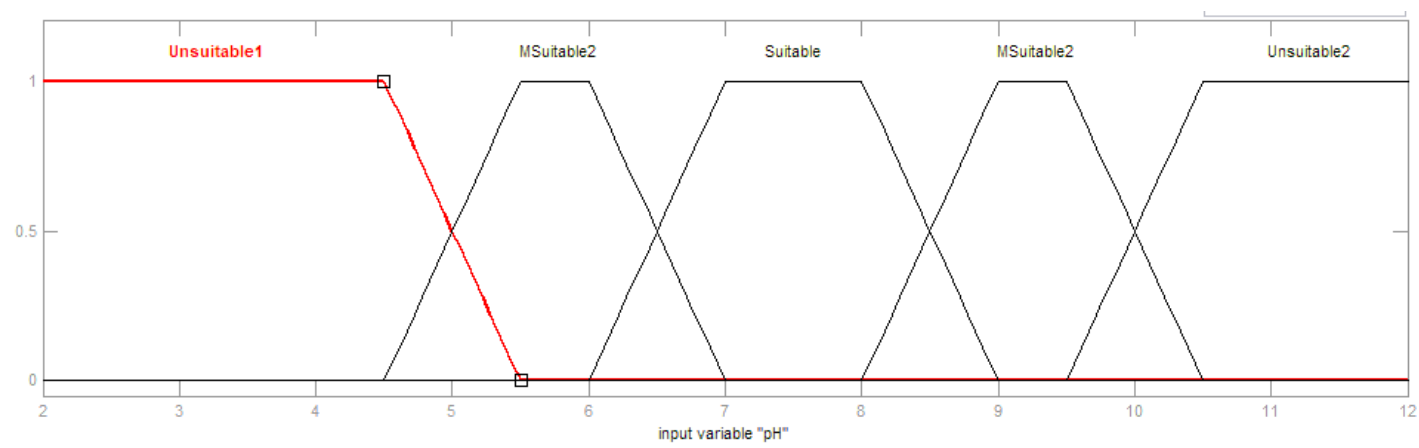

Figure 2a: Illustrative Membership function for 'pH' Input Variables

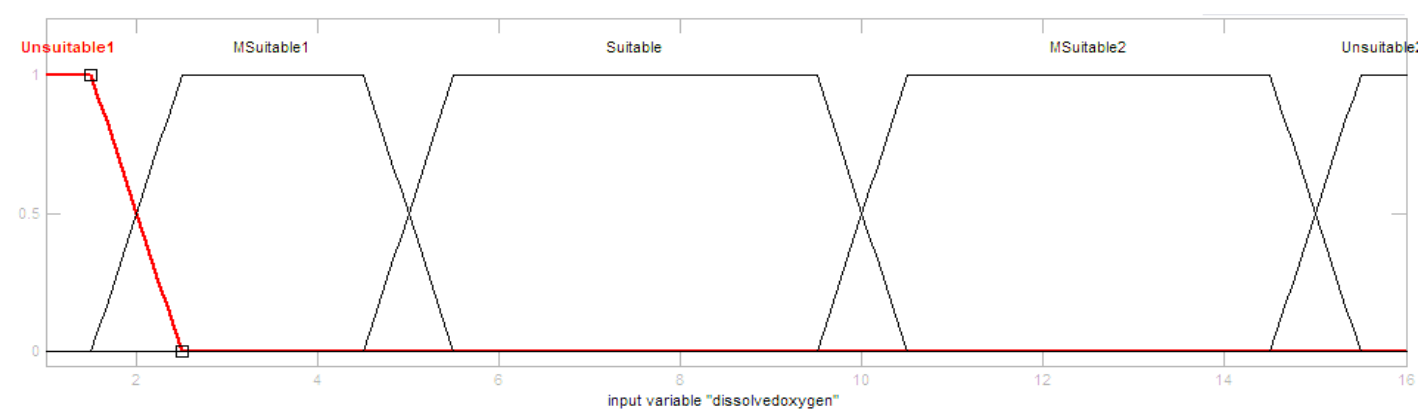

Figure 2b: Illustrative Membership Function for 'Dissolved Oxygen' Variable 


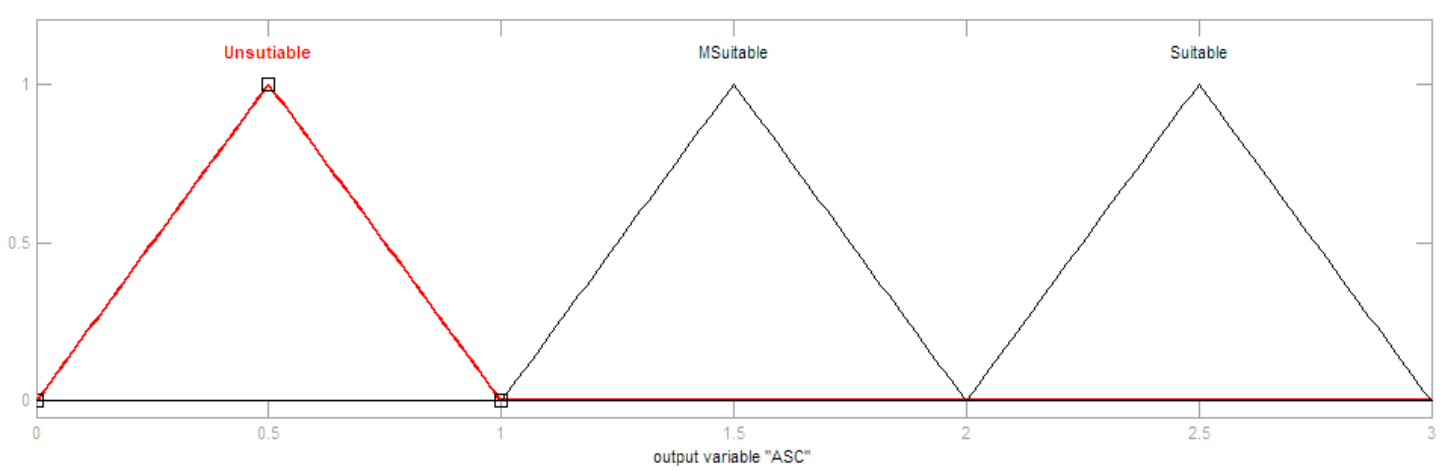

Figure 3: Membership Function for Output Variable

\section{Step 4: Aggregation}

Since decisions are based on the testing of all of the rules in the model, rules must be combined in order to make decisions. In this model, the aggregation was performed by using union (maximum) operator.

\section{Step 5: Defuzzification}

Among the various defuzzification mechanisms by which central sums, central most region first maximum, and the maximum center of gravity (COG) method are most commonly used, classification programs are used because it is well known for having no root-mean-square error and better sustained performance (Kim et al., 2001). This model COG method is used for defuzzification to convert fuzzy output to an exact number. The centroid of the aggregated area was defined $A F C=\sum_{i=1}^{n} a_{i} c_{i} / \sum_{i=1}^{n} a_{i}$ where $a_{1} a_{2}, \ldots \ldots, a_{n}$ be the areas of the truncated triangular areas under the aggregated function and $c_{1}, c_{2}, \ldots \ldots ., c_{n}$ be the coordinates of their center on the $\mathrm{x}$-axis, $\mathrm{n}$ is the number of areas and AFC is the location of the centriod of the total areas. The location of COG determines the classification of optimal location for aquaculture farming development.

\section{FUZZY MODEL IMPLEMENTATION AND ITS RESULTS}

The proposed fuzzy model was implemented as a fuzzy rule-based tool using MATLAB (Version R13.a). In this study, fuzzy inference was performed based on Mamdani's inference method which uses minimum (MIN) operator in the implication process and maximum (MAX) operator in the aggregation process. The FIS editor of the developed program is shown in Figure 4. 


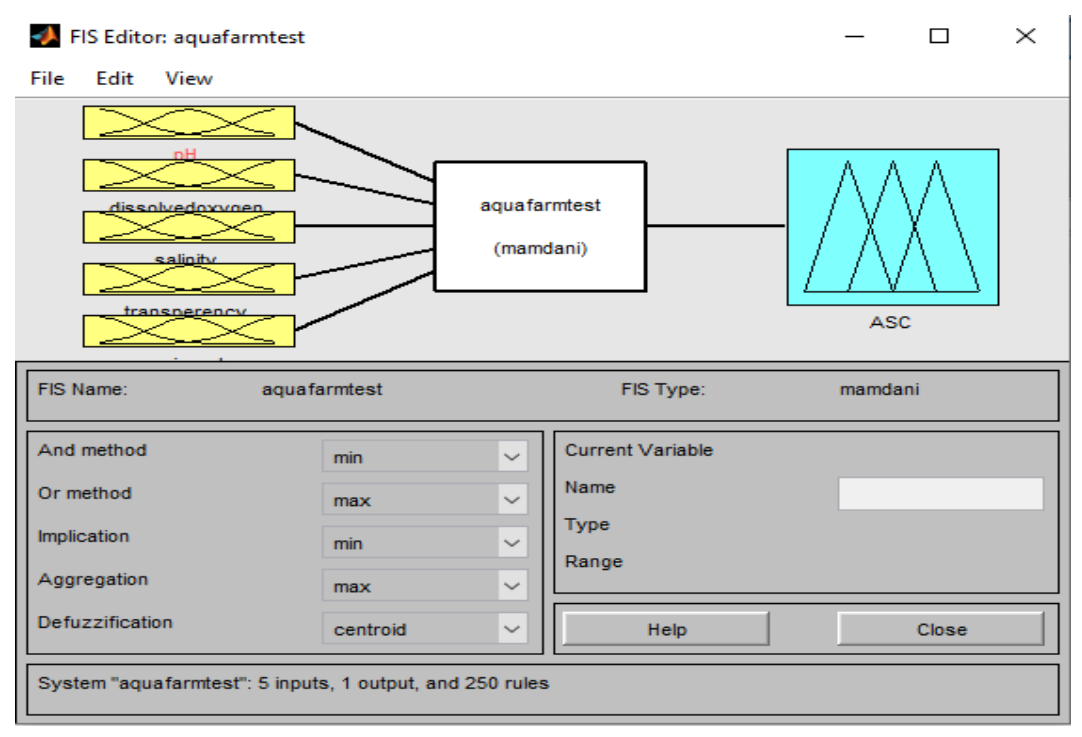

Figure 4: Schematic Implementation of Proposed Fuzzy Model

Then, the membership functions for each input variable (Fig. 5) and output variable were generated using the membership function editor in the Matlab fuzzy logic toolbox. The rule editor was used for generating the 3125 fuzzy ifthen rules in Matlab. By using a numerical example illustration, the working procedure of the proposed model can be explained as follows: Suppose that information concerning the input variables are expressed numerically as follows: $\mathrm{pH}=$ 8.3 , dissolved oxygen $=6.5$, salinity $=12$, transparency $=50$, and organic carbon $=2.1$.

- At the first step, fuzzification yields the following fuzzy inputs for the next step in the inference process:

Input 1: $\mathrm{pH}$ is suitable with membership degree 0.6 or moderately suitable2 with membership degree 0.25

Input 2: Dissolved oxygen is suitable with membership degree 1

Input 3: Salinity is moderately suitable1 with membership degree 1

Input 4: Transparency is suitable with membership degree 1

Input 5: Organic carbon is suitable with membership degree 0.3 ; moderately suitable 2 with membership degree 0.85 ; or unsuitable 2 with membership degree 0.3

- Then, the fuzzified values were used by the proposed model to activate appropriate rules and calculate weights factor as follows: Activation rules: 26, 28, 30, 1276, 1278, 1280 and Weight factors as per: $\quad$ Rule 26 as MIN $(\{0.6,1,1,1,0.3\})=0.3$; Rule 28 as MIN $(\{0.6,1,1,1,0.85\})=0.6$; Rule 30 as MIN $(\{0.6,1,1,1,0.3\})=0.3$; Rule 1276 as $\operatorname{MIN}(\{0.25,1,1,1,0.3\})=0.25$; Rule 1278 as $\operatorname{MIN}(\{0.25,1,1,1,0.85\})=0.25$ and Rule 1280 as MIN $(\{0.25,1,1,1,0.3\})=0.25$

- At this point, based on the weight, the fuzzy result of each rule is calculated using the THOUSANDS operator, including in the fuzzy output using the MAX operator as MAX $(\{0.3,0.6,0.3,0.25,0.25,025\})$

At last, the model shows defuzzification of the combined fuzzy output to provide short \& Clear output value 2.22 (Fig. 6) and its corresponding linguistic value suitable as the output of the classification. Similar processes were carried out by the model for the crisp values in the dataset. The result of this procedure for the test dataset is summarized in Table 4 . 


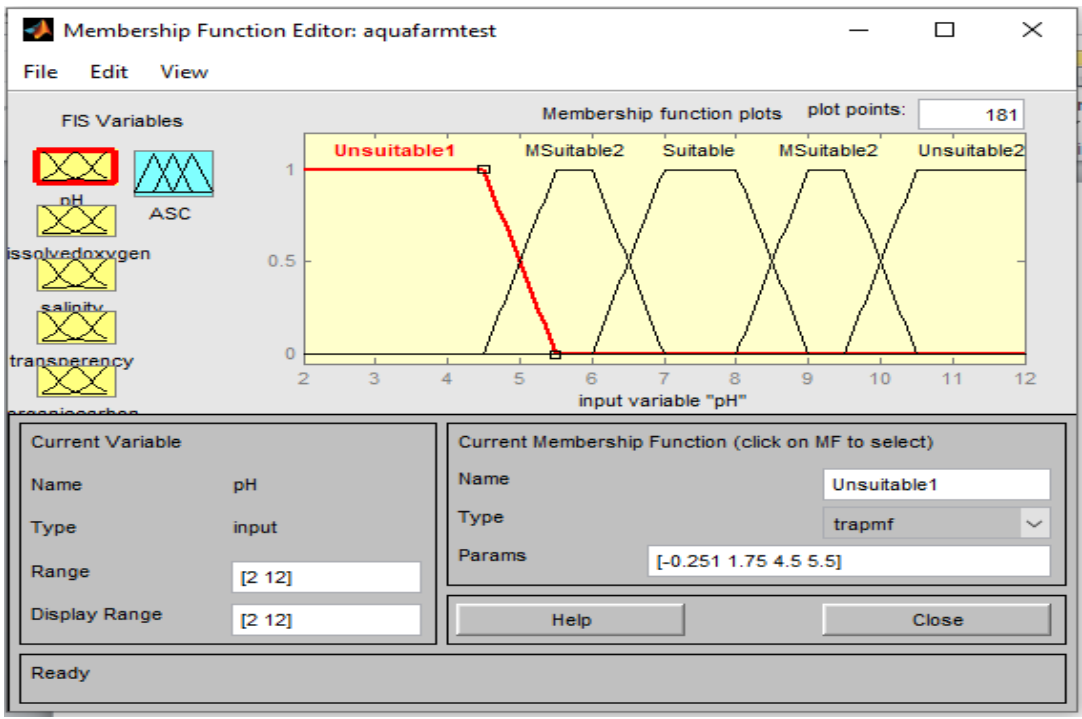

Figure 5: One of the Example Editor Toolbox for 'pH' Input Variable

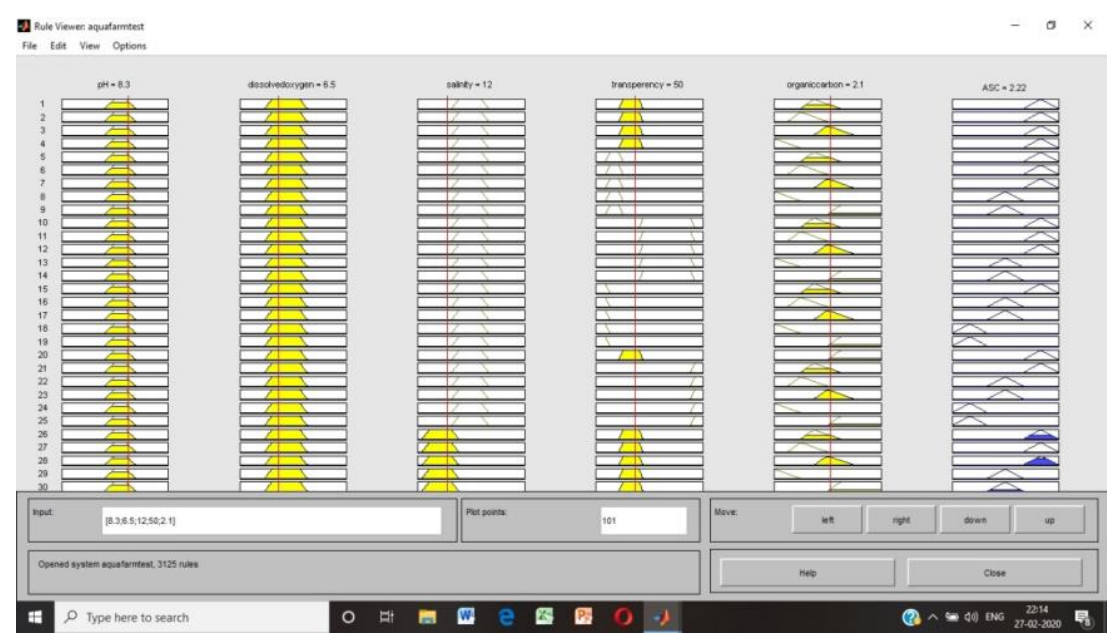

Figure 6: Fuzzy Model Output for Suitable Classification of Site

\section{CONCLUSIONS}

The developed fuzzy logic based data conglomeration model in multi sensor system for optimizing aquafarms leads to use effective utilization of various resources like water, land, infrastructure facilities, support and inputs and hence becomes a cost effective system for the expected yield. The fuzzy controller designed in our study efficiently monitors water depth, water temperature, salinity, water hardness, water alkalinity and other important parameters sent from wireless sensor network which is deployed in a field to be monitored to control the culture practices. The results show that the application is correct and reasonable and enables a user to precisely acquire the culture requirement information. The fuzzy model can be further extended to utilize the modified k-means algorithm for further classification into different categories within the suitable, moderate or unsuitable categories. Other potential areas for further scope include the development of online decision making models using the same procedure but through web-based software techniques. This would be useful to decision makers across the world. The model can be further extended to include more environmental, water and soil parameters. 


\section{REFERENCES}

1. Boyd, C.E. \& Clay, J.W. (1998). Shrimp aquaculture and the environment Scientific American., 278: 58-65.

2. P. Senthil Kumar, B. Sridhar Babu \& V. Sugumaran, "Comparative Modeling on Surface Roughness for Roller Burnishing Process using Fuzzy Logic”, International Journal of Mechanical and Production Engineering Research and Development (IJMPERD), Vol. 8, Issue 1, pp, 43-64

3. Center, B. \& Verma, B. (1998). Fuzzy logic for biological and agricultural systems, Artificial Intelligence Review, 12: 213225.

4. B. Amudhambigai, A. Neeraja \& R. Roseleen Nithiya, "A Study on the Advent and Wield of Technology using Fuzzy Logic”, International Journal of Human Resource Management and Research (IJHRMR), Vol. 8, Special Issue, pp, 127-132

5. CIBA (2018). Recent advances in soil and water management in brackishwater aquaculture CIBA TM-series No. 8, Central Institute of Brackishwater Aquaculture, pp. 136.

6. Deepti Sharma \& Abhay Sharma, “Dynamic Spectrum Sharing in Cognitive Radio Using Fuzzy Logic System”, International Journal of Electronics and Communication Engineering (IJECE), Vol. 4, Issue 1, pp, 1-12

7. Johannes, A. R., Setnes, M. \& Abonyi, J. (2003). Learning fuzzy classification rules from labeled data. Information Science, 150: 77-93.

8. Kim, S., Kim, K, Lee, J. \& Cho, D. (2001). Application of Fuzzy Logic to Vehicle Classification Algorithm in Loop/PiezoSensor Fusion Systems. Asian Journal of Control, 3: 64-68.

9. G. Venkateswarlu, C. H. Jhansi \& P. S. Raju, "Fuzzy Logic Based Maximum Power Point Tracking Technique GOR Partially Shaded Photovoltaic System", International Journal of Electrical and Electronics Engineering (IJEEE) ISSN(P): 2278-9944; ISSN(E): 2278-9952 Vol. 3, Issue 1, pp, 49-60

10. Mahalakshmi, P. \& Ganesan, K. (2009). Mahalanobis Taguchi System based variables selection for shrimp aquaculture development. Computer and Electronics in Agriculture, 65(2): 192-197.

11. Mazloumzadeh, S. M., Shamsi, M. \& Nezamabadi-pour, H. (2010). Fuzzy logic to classify date palm trees based on some physical properties related to precision agriculture. Precision Agriculture, 11: 258-273.

12. McKindsey, C.W., Thetmeyer, H, Landry, T. \& Silvert, W. (2006). Review of recent carrying capacity models for bivalve culture and recommendations for research and management. Aquaculture, 26: 451-462.

13. Pedrycz, W. (2001). Fuzzy equalization in the construction of fuzzy sets. Fuzzy Set System, 119: 329-335.

14. Singh, J., Singh, N. \& Sharma, J. K. (2006). Fuzzy modeling and identification of intelligent control for refrigeration compressor. Journal of Scientific \& Industrial Research, 65: 22-30.

15. Tarunamulia. (2008). Application of Fuzzy Logic, GIS and Remote Sensing to the Assessment of Environmental Factors for Extensive Brackishwater Aquaculture in Indonesia. Unpublished Ph.D thesis, School of Biological, Earth \& Environmental Sciences, The University of New South Wales Sydney, NSW 2052, Australia, pp. 206.

Table 1: Variables and its Suitability Range

\begin{tabular}{|c|l|l|l|}
\hline \multirow{2}{*}{ Variables } & \multicolumn{2}{|c|}{ Range } \\
\cline { 2 - 4 } & \multicolumn{1}{|c|}{ Suitable } & \multicolumn{1}{c|}{ Moderately suitable } & Unsuitable \\
\hline $\mathrm{pH}(\mathrm{ppt})$ & {$[6.5,8.5]$} & $(5,6.5)$ or $(8.5,10)$ & $\leq 5.0$ or $\geq 10.0$ \\
\hline Dissolved Oxygen $(\mathrm{mg} / \mathrm{litre})$ & {$[5,10]$} & $(2,5)$ or $(10,15)$ & $\leq 2$ or $\geq 15$ \\
\hline Salinity & {$[15,25]$} & $(5,15)$ or $(25,35)$ & $\leq 5$ or $\geq 35$ \\
\hline Transparency $(\mathrm{cm})$ & {$[30,60]$} & $(15,30)$ or $(60,120)$ & $\leq 15$ or $\geq 120$ \\
\hline Organic carbon $(\%)$ & {$[1.5,2]$} & $(0.5,1.5)$ or $(2,2.5)$ & $\leq 0.5$ or $>2.5$ \\
\hline
\end{tabular}


Table 2: Linguistic Variables of the Input Variables

\begin{tabular}{|l|l|l|l|l|l|}
\hline \multicolumn{1}{|c|}{ Variables } & \multicolumn{1}{|c|}{ Suitable } & $\begin{array}{c}\text { Unsuitabl } \\
\text { e 1 }\end{array}$ & \multicolumn{1}{|c|}{ Usuitable2 } & \multicolumn{1}{c|}{$\begin{array}{c}\text { Moderately } \\
\text { Suitable 1 }\end{array}$} & $\begin{array}{c}\text { Moderately } \\
\text { Suitable 2 }\end{array}$ \\
\hline $\mathrm{pH}$ & $6<7<8<9$ & $4.5<5.5$ & $9.5<10.5$ & $4.5<5.5<6<7$ & $\begin{array}{l}8<9<9.5 \\
<10.5\end{array}$ \\
\hline Dissolved oxygen & $4.5<5.5<9.5<10.5$ & $1.5<2.5$ & $14.5<15.5$ & $1.5<2.5<14.5<15.5$ & $\begin{array}{l}9.5<10.5<14.5 \\
<15.5\end{array}$ \\
\hline Salinity & $13.5<16<23.5<26$ & $3.5<6$ & $33.5<35.5$ & $3.5<6<13.5<16$ & $\begin{array}{l}23.5<26<33.5 \\
<36\end{array}$ \\
\hline Transparency & $30<35.5<55.5<60.5$ & $13<19$ & $120<125$ & $13<19<30<35.5$ & $\begin{array}{l}55.5<60.5<120 \\
<125\end{array}$ \\
\hline Organic carbon & $1<1.5<1.5<2.5$ & $0<1$ & $2<2.5$ & $0.5<1<1<2$ & $1.5<2<2<3$ \\
\hline
\end{tabular}

Table 3: Linguistic Variables of the Output Variable

\begin{tabular}{|c|c|c|c|}
\hline Variables & Unsuitable & $\begin{array}{c}\text { Moderately } \\
\text { Suitable }\end{array}$ & Suitable \\
\hline AFC & $0<0.5<1$ & $1<1.5<2$ & $2<2.5<3$ \\
\hline
\end{tabular}

Table 4: Outputs of the Fuzzy Model for Dataset

\begin{tabular}{|c|c|c|c|c|c|c|c|c|}
\hline \multirow{2}{*}{$\begin{array}{c}\text { Sites or } \\
\text { Aqua } \\
\text { Farms }\end{array}$} & $\mathbf{p H}$ & $\begin{array}{c}\text { Dissolved } \\
\text { Oxygen }\end{array}$ & Salinity & Transparency & $\begin{array}{c}\text { Organic } \\
\text { carbon }\end{array}$ & Active Rules & $\begin{array}{c}\text { Input (Dataset) } \\
\text { Crisp Value }\end{array}$ & $\begin{array}{c}\text { Classification } \\
\text { by model* }\end{array}$ \\
\hline S1 & 8.3 & 6.5 & 12 & 50 & 2.1 & $\begin{array}{c}26,28,30,127 \\
6,1278,1280\end{array}$ & 2.22 & $\mathrm{~S}$ \\
\hline S2 & 7.9 & 12 & 18 & 45 & 1.3 & 251,252 & 2.5 & $\mathrm{~S}$ \\
\hline S3 & 15 & 0.5 & 7.5 & 53 & 0.8 & 2902,2904 & 0.5 & $\mathrm{U}$ \\
\hline S4 & 5.8 & 8.5 & 50 & 82 & 1.7 & $736,737,738$ & 1.5 & $\mathrm{M}$ \\
\hline S5 & 22 & 18 & 31 & 75 & 4.5 & 3065 & 0.5 & $\mathrm{U}$ \\
\hline
\end{tabular}

*S-Suitable; M- Moderate; U- Unsuitable 